\title{
Celso Furtado (1920-2004)
}

\section{Ein Nachruf von Theotonio dos Santos}

Celso Furtados schneller und unerwarteter Tod hinterlässt eine große Leere im kritischen Denken Brasiliens und auf internationaler Ebene. Das Ansinnen, ihn für den Nobelpreis 2004 vorzuschlagen, mobilisierte eine beeindruckende Unterstützung in Brasilien, Lateinamerika und in der ganzen Welt. Doch die konservative Jury hat die Gelegenheit verpasst, die Glaubwürdigkeit des Nobelpreises zu erhöhen und hat statt dessen den Ruf des Preises durch eine klare Präferenz für visionslose und konservative Ökonomen weiter beschädigt.

Es ist absurd, dass das lebendige ökonomische Denken, das sich in Lateinamerika insbesondere unter dem Einfluss von Raúl Prebisch und der Wirtschaftskommission der Vereinten Nationen für Lateinamerika (CEPAL) entwickelt hat, weiterhin isoliert vom Mainstream der Wirtschaftswissenschaften bleibt. Stets hat sich Celso Furtado der Aufgabe gewidmet, die Irrtümer des konservativen Denkens aufzudecken. Seine Kritik galt Positionen, die die internationale Arbeitsteilung festschreiben oder gar verklären, und die Rolle der peripheren und unterentwikkelten Staaten auf eine subalterne Funktion der Zulieferung für die produktive Weiterverarbeitung und den Konsum in den Industrieländern beschränkt.

Celso Furtado zeigte die Verschlechterung der realen Austauschverhältnisse zwischen dem Zentrum und der Peripherie auf (Konzepte, die von der CEPAL entwickelt wurden) und wies auf die ungleiche Entwicklung des technologischen Fortschritts hin. Ihm war klar: Ohne eine fundamentale politische und ökonomische Wende würden sich die internationalen Wirtschaftsbeziehungen weiter in Richtung einer zunehmenden globalen Ungleichheit entwickeln und gerade nicht hin zu einer Konvergenz, wie dies im Rahmen des Gesetzes der komparativen Kostenvorteile behauptet und von der orthodoxen Wirtschaftswissenschaft verbreitet und versprochen wird.

Die Industrialisierung der peripheren Staaten ist nur mit Hilfe einer Politik der staatlichen Planung möglich, die von Celso Furtado mit großem Können systematisch untersucht wurde. Er zeigte auch, dass „Unterentwicklung“ nicht einfach nur ein Zeichen ökonomischer Rückständigkeit ist, sondern eigenständige Gesetzmäßigkeiten besitzt. Sie zu erforschen, bedarf eines eigenen theoretischen Ansatzes, zu dessen Entwicklung Furtado entscheidend beitrug.

Neben seinen Beiträgen zur Theorie der Unterentwicklung und der Planung, beschäftigte sich Celso Furtado mit der Wirtschaftsgeschichte Brasiliens und 
Lateinamerikas. Seine Bücher über die wirtschaftliche Entwicklung Brasiliens und Lateinamerikas hatten, wie Fernand Braudel anerkannte, einen starken Einfluss auf die Annales-Schule.

Celso Furtado machte ebenfalls bedeutende Beiträge zur Analyse der Weltwirtschaft. Bereits in den 60er Jahren nahm er in seinen Arbeiten wichtige Ergebnisse der Globalisierungsdiskussion über die Auswirkungen multinationaler Unternehmen auf die kapitalistische Wirtschaft vorweg.

Er antizipierte auch die negativen Effekte, die die Dominanz bestimmter Denkschulen auf die Situation von Schwellenländern wie Brasilien haben würde. In den vergangenen Jahren ist sein Werk zu einem der wichtigsten Bezugspunkte der brasilianischen Opposition geworden, die bei den letzten Wahlen eine überwältigender Mehrheit erhielt und die Regierung stellt. Dabei handelt es sich nicht allein um eine politische Debatte, sondern auch um eine theoretische Diskussion über die Ausrichtung der Wirtschaftswissenschaften; Celso Furtado hat eine breite methodische Reflexion über den Sinn der Wirtschaftswissenschaften angestoßen. Vor allem wendete sich seine Kritik gegen den Ansatz der ökonomischen Theorie, der sich den gesellschaftlichen Problemen eher von der Seite des Weltbildes der Physik des späten 19. Jahrhunderts aus nähert, als aus der Perspektive der modernen Sozialwissenschaften.

Celso Furtado hat damit das sozialwissenschaftliche Denken Lateinamerikas in bedeutendem Maße geprägt und wichtige Beiträge zur sozialwissenschaftlichen Theorie und Methodik geliefert. Im Bereich der Theoriebildung leistete er unter anderem Entscheidendes zur Entwicklung des historischen Strukturalismus. In der Anwendung dieses Ansatzes widmete er sich der Geschichte der USA, der Entwicklung des Kapitalismus in Europa und dessen weltweiten Auswirkungen, sowie der Dynamik der lateinamerikanischen Wirtschaft. Und da seine Forschung in der Praxis stets auch einen interdisziplinären Anspruch beinhaltete, wurden seine Bücher für die Ausbildung von Wirtschafts- und Sozialwissenschaftlern in ganz Lateinamerika unentbehrlich.

Aber das Werk Celso Furtados beschränkt sich nicht allein auf den Bereich der Wissenschaft. Sein Wirken als Person des öffentlichen Lebens begann Ende der fünfziger Jahre mit der Gründung des Entwicklungsamtes im Nordosten Brasiliens (SUDENE). Er zeigte die Bedeutung der Regionalplanung auf, die sich dort der Bekämpfung des Elends in einer ganzen Region Brasiliens widmete. Ausgehend von den Erfahrungen der Tennessee Vale Authority in den USA und dem Planungsprogramm für Sizilien ebnete Celso Furtado den Weg zur Rettung des brasilianischen Nordostens. Eine Region, mit der sich auch Josué de Castro in seinem Buch Geopolitik des Hungers eingehend beschäftigte. Der Arzt, Geograph und Soziologe aus Pernambuco erregte mit seiner Arbeit weltweit Aufmerksamkeit und beeinflusste das Ernährungsprogramm „Zero Fome“ des neuen brasilianischen Präsidenten Lula, das derzeit leider immer mehr in Vergessenheit zu geraten droht. 
Celso Furtados Erfolg in der SUDENE führte unter Präsident Goulard zur Einrichtung des Planungsministeriums, von wo aus Furtado seine theoretischen Erkenntnisse in praktische Maßnahmen umsetzen konnte. Diese Arbeit wurde 1964 durch den Militärputsch unterbrochen. Für zwei Jahrzehnte wurde das sozialwissenschaftliche Denken in Brasilien einem kolonialen Diktat unterworfen, von dem es sich bis heute nicht vollständig erholt hat. Lange Zeit verbrachte Celso Furtado im Exil. Nach dem Ende der Diktatur und den Präsidentschaftswahlen von 1984 wurde er Kulturminister und später Botschafter Brasiliens bei der Europäischen Union in Brüssel. In diesen Jahren nahm er starken Einfluss auf die Annäherung und Zusammenarbeit zwischen Brasilien und Argentinien, was später zur Gründung des MERCOSUR führen sollte.

Der Einfluss Celso Furtados auf das sozialwissenschaftliche Denken ist kaum hoch genug einzuschätzen. Seine Bedeutung wird noch zunehmen, wenn die brasilianische Bevölkerung und die Menschen in Lateinamerika eine alternative Wirtschaftspolitik für diese Region fordern. Solange die Verantwortlichen für die derzeitige wirtschaftliche und soziale Katastrophe behaupten, dass es nur eine „einzig mögliche“ Wirtschaftspolitik gäbe, wird das Werk von Celso Furtado eine wichtige Waffe im Kampf gegen genau diesen Mythos sein. Wenn wir die Debatte über ein nachhaltiges Wirtschaftswachstum, über die Verteilung von Einkommen und Fragen der sozialen Gleichheit wieder aufnehmen, wird das Werk von Celso Furtado für uns von großem Nutzen sein. Denn dann werden wir in seinen Arbeiten über Entwicklung, Planung und politische Ökonomie ein fundamentales Werkzeug für die Ausbildung einer neuen Generation von Ökonomen in Brasilien, in Lateinamerika und in der ganzen Welt finden.

Aus dem brasilianischen Portugiesisch übersetzt von Verena Holzer 


\section{Thema: Krieg und Frieden}

Bernhard Sutor

\section{Vom gerechten Krieg zum gerechten Frieden?}

\section{Stationen und Chancen eines geschichtlichen Lernprozesses}

Die Rede vom „gerechten Krieg“ provoziert heute Abwehr. Krieg und Gerechtigkeit passen für uns nicht zusammen. Friede und Ungerechtigkeit aber auch nicht - und der Friede muss geschützt werden.

Das neuzeitliche Völkerrecht ersetzte die alte, im Rahmen einer Friedensethik entwickelte Denkfigur vom gerechten Krieg durch das allgemeine Recht des Staates, Krieg zu führen. Es ging nicht mehr um Moral, sondern um Staatsräson. Die Katastrophe zweier Weltkriege hat dieses Konzept ad absurdum geführt.

Nach 1945 sollten die Vereinten Nationen eine Ordnung des Weltfriedens sichern. Der Kalte Krieg hat dem enge Grenzen gesetzt, aber nach dessen Ende sind Kriege, vor allem Bürgerkriege, noch zahlreicher geworden. Gibt es Chancen, den Krieg durch gemeinsame Anstrengungen der Staaten dauerhaft zu überwinden?

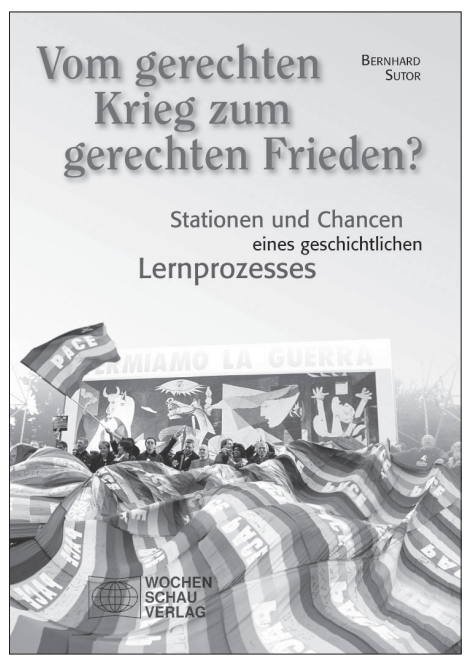

ISBN 3-89974152-8, 208 S., € 14,80

Die vorliegende Schrift geht dieser Frage nach, indem sie die Entwicklung von Ethik und Völkerrecht zu Krieg und Frieden von der Antike bis in unsere Zeit nachzeichnet.

\section{www.wochenschau.verlag.de}




\section{Dagmar Vinz}

\section{Nachhaltiger Konsum und Ernährung Private KonsumentInnen zwischen Abhängigkeit und Empowerment}

Mit der Kampagne „Echt gerecht - clever kaufen“ wirbt das Bundesministerium für Verbraucherschutz, Ernährung und Landwirtschaft (BMVEL) in Kooperation mit anderen Akteuren für eine nachhaltige Ernährungsweise, insbesondere durch die Veränderung von Konsummustern mit der Entscheidung für fair produzierte Produkte. Zum Auftakt der Kampagne unterstrich die Ministerin Renate Künast die hohe Relevanz der Entscheidungsmacht von VerbraucherInnen im Nachhaltigkeitsprozess mit den Worten: „Mit unseren Kaufentscheidungen beeinflussen wir jeden Tag aktiv nicht nur unser eigenes Leben, sondern das vieler anderer in anderen Teilen der Erde und das Leben nachfolgender Generationen“ (BMVEL 2004). Damit wird den KonsumentInnen ein hohes Maß an Gestaltungsmacht zugesprochen: Sie sind es, die über die Zukunft der Ernährung und über den Grad der Nachhaltigkeit in Landwirtschaft und Ernährung entscheiden. Im Kontext der Politik für eine Agrarwende waren KonsumentInnen immer wieder gefordert, mehr Geld für Nahrungsmittel auszugeben, bei deren Kauf Nachhaltigkeitskriterien zu berücksichtigen und den Anteil der Ausgaben für Lebensmittel im Haushaltsbudget zu erhöhen. Dabei hängt die Zukunft der Agrarwende zum einen sicher davon ab, ob „eine ökologische Wende in der Landwirtschaft auf die KonsumentInnen zählen" (Brunner 2004) kann. Zum anderen wird in der Nachhaltigkeitsdebatte vor einer Überhöhung der Gestaltungsmacht privater KonsumentInnen gewarnt. Um mit Anforderungen an ökologisch und politisch korrekten Einkauf und gesunde Ernährung nicht einer „Privatisierung und Feminisierung der Umweltverantwortung“ (Weller 2004) Vorschub zu leisten, ist es notwendig zu analysieren, wie der Konsum in das gesamte Ernährungssystem eingebettet ist und welche Einfluss- und Gestaltungsmöglichkeiten die KonsumentInnen im Vergleich zu den anderen Akteuren auf den verschiedenen Ebenen des Ernährungssystems - Produktion, Verarbeitung, Handel und Konsum haben. 


\section{Konsumwandel im Ernährungssystem}

Als fordistisch ist das Ernährungssystem ${ }^{1}$ nach 1945 bezeichnet worden, in welchem die Massenproduktion erfolgreich an den Massenkonsum von Nahrungsmitteln gebunden ist (Goodman/Redclift 1991: 87ff; Fine/Heasman et al. 1996; Friedmann 1999). Dem Ziel verpflichtet, die Bevölkerung mit einer ausreichenden Menge an Lebensmitteln zu niedrigen Preisen zu versorgen, wurde mit einem hohen Zufluss von Energie, Material und Kapital die Mechanisierung der Landwirtschaft, die Intensivierung der Produktion durch den Einsatz von Dünge- und Pflanzenschutzmitteln und die Vergrößerung und Spezialisierung der landwirtschaftlichen Betriebe durchgesetzt. Damit etablierte sich ein System, das bis heute nur durch hohe Subventionen, Marktabschottung gegenüber Importen und besondere Gesetze existieren kann (Brunner 2001: 210). Im Zusammenhang mit veränderten Beschäftigungs- und Einkommensstrukturen in den privaten Haushalten der nördlichen Industrieländer wiederum, die aus der zunehmenden Beschäftigung im Dienstleistungsbereich und der Herausbildung einer neuen kaufkräftigen Elite resultiert, ist seit den 1970er Jahren eine neue Nachfrage nach handwerklich verarbeiteten oder exotischen Nahrungsmitteln entstanden (Friedland 1994; Friedland 1994a: 213ff; Friedmann 1999). Dementsprechend wird die Massenproduktion für stärker differenzierte Märkte vorangetrieben, und in diesem Zusammenhang kennzeichnet ein paralleler Prozess von Globalisierung und Regionalisierung den Weg in ein globales postfordistisches Ernährungssystem.

Als Voraussetzung für die Koppelung von Massenproduktion und -konsum werden mit zunehmender Verarbeitungstiefe aus verderblichen Nahrungsmitteln dauerhafte Konsumgüter, ebenso wird der massenhafte Vertrieb durch die Ausweitung des Prinzips der Selbstbedienung in modernen Supermärkten revolutioniert. Im Rahmen dieser Entwicklung gewinnen Lebensmittelindustrie und Einzelhandel nicht nur gegenüber der Landwirtschaft an Relevanz und Marktmacht. Auch die Rolle privater Haushalte verändert sich tiefgreifend im Zuge der zunehmenden Verlagerung von Arbeiten aus den Haushalten an den Markt und durch die räumliche und zeitliche Entkoppelung von Produktion und Konsum im Kontext von Globalisierungsprozessen. Produktion und Konsum sind dabei in komplexer Weise verflochten, so dass keine Ebene des Ernährungssystems als dominant oder determinierend zu gelten kann.

1 Der Begriff des Ernährungssystems wird gewählt, um das Zusammenspiel zwischen den verschiedenen Ebenen - Produktion, Verarbeitung, Handel, Konsum - zu erfassen (Tansey/ Worsley 1995). Das Konzept des Ernährungssystems verknüpft drei Bereiche: auf biologischer Ebene die sozial-ökologischen Prozesse, die der Produktion von Nahrungsmitteln zugrunde liegen und deren ökologische Nachhaltigkeit; auf ökonomischer und politischer Ebene die Macht und Kontrolle verschiedener Akteursgruppen über die verschiedenen Teile des Ernährungssystems; auf sozialer und kultureller Ebene die persönlichen Bindungen, Gemeinschaftswerte und kulturellen Traditionen, die den Konsum von Lebensmitteln charakterisieren. 


\subsection{Massenproduktion und -konsum}

Grundlegend für die zuvor nicht gekannte Entwicklung des Massenkonsums seit den 1950er Jahren ist der starke Anstieg der Produktivität nicht nur im Bereich der industriellen Produktion, sondern auch in der Landwirtschaft. Dabei werden in der Massentierhaltung oder beim Gemüseanbau in Gewächshäusern arbeitsorganisatorische Ansätze industrieller Massenproduktion auf die Bauernhöfe übertragen. Im Bereich der intensiven Tierzucht basiert die Massenproduktion auf einer beispiellosen Produktivitätssteigerung durch die Beschleunigung und Kontrolle der tier- und pflanzeneigenen Rhythmen mit Hormonspritzen, durch die Fütterung mit Kraftfutter oder die Behandlung mit Antibiotika (Schneider 1999). Die intensive Tierzucht in spezialisierten Betrieben ermöglicht dank gesunkener Fleischpreise dessen Massenkonsum und hat so die tradierten Wertigkeiten der Nahrungsmittel auf den Kopf gestellt: „Infolge der gleichzeitigen Entwicklung des Automobilismus wurde das Fleisch des traditionellen Resteverwertungstiers ärmerer Leute, des Pferds, teurer als Rindfleisch. Da das Schwein sich leichter in Massen produzieren lässt als das Rind, verkehrte sich auch deren Preisverhältnis (...) Schließlich sank der Hühnerpreis ins Bodenlose, während vordem das Huhn ein relativ teures Tier war" (Schwendter 1995: 235).

Auch Fische und Schalentiere sind zu Produkten aus der Massentierhaltung geworden: Waren Lachs und Shrimps noch vor wenigen Jahren kostspielige Spezialitäten, die sich nur eine Minderheit leisten konnte, so sind sie heute zu günstigen Preisen im Tiefkühlregal erhältlich und „in aller Munde“ (KallerDietrich 2004). Ihr Preisverfall resultiert daraus, dass mit der Verbreitung von Aquakulturen die Prinzipien der Massenproduktion vom Land aufs Wasser übertragen werden (Prahl/Setzwein 1999: 251ff). Zwar werden die norwegischen Zuchtlachse nie nach Alaska schwimmen und dort ablaichen, dafür werden sie mit Fischmehl, das aus Peru stammen kann, gemästet und unter dem Label „Wildlachs“ für eine breite Schicht von KonsumentInnen erschwinglich (Kaller-Dietrich 2004). Massenproduktion von Nahrungsmitteln ist daher mit einer sinkenden sozialen Differenzierung des Konsums und einer Homogenisierung der Ernährungsgewohnheiten verknüpft. Dabei folgt der erhöhte Fleischkonsum nicht rein reaktiv auf die veränderten Angebote der Massenproduktion, sondern geht auf eine europäische Esstradition zurück, in welcher der Fleischverzehr mit Wohlstand assoziiert wird und besonders in der Esskultur der Nachkriegszeit als essentieller Bestandteil einer „richtigen Mahlzeit" galt. Wenn der Konsum von Lachs und Schalentieren hingegen seit Mitte der 1980er Jahre ausgeweitet wurde, so passt dies zur stärkeren Orientierung an gesundheits- und kalorienbewussten Ernährungsleitbildern und der entsprechenden Vermarktung dieser Produkte als fettarm bzw. reich an sog. „guten“ Omega-3-Fettsäuren. Die Ausweitung der Nachfrage hat wiederum Anreize für die Ausweitung von intensiver Tierzucht und den dafür notwendigen Futtermit- 
telanbau bzw. für die Vermehrung von Aquakulturen bewirkt - mit den bekannten negativen Folgewirkungen für die Nachhaltigkeit des Ernährungssystems. ${ }^{2}$

\subsection{Konsumwandel und Lebensmittelverarbeitung}

Für die Koppelung von Massenfertigung und -konsum ist die Verarbeitung von schnell verderblichen und wenig haltbaren Nahrungsmitteln zu dauerhaften Konsumgütern eine elementare Voraussetzung. Der Verfall der Nahrungsmittel wird nicht nur herausgezögert, damit sie Transportwege unbeschadet überstehen, sondern auch, damit ihre Lebensdauer im Regal verlängert wird und das Produkt nach seinem Kauf nicht zu schnell verdirbt. Im Zuge der Industrialisierung der Landwirtschaft sind zeitaufwendige Methoden wie das Lufttrocknen, Räuchern, Salzen, Säuern oder in Honig Einlegen von energetisch aufwendigen industriellen Verfahren wie der Tiefkühlung, der Aufbewahrung in Konserven oder der Beigabe chemischer Konservierungsstoffe verdrängt worden (König 1998). Im Sog der zunehmenden Verarbeitungstiefe verändern sich Ausgangsprodukte „vom lebenden zum gerupften, zerteilten, bis zum in Dosen erhältlichen vorgekochten Huhn und schließlich zu portionsfertig aufbereiteten, fertig panierten tiefgekühlten Hähnchenteilen" (LeichtEckardt 1999: 196). Neue Fertiggerichte werden erfunden und durchgesetzt und in den Küchen der Food-Designer auf Lyfe-Style und Lebensgewohnheit hin angepasst. Mit ihrem Vertrieb gewinnen transnationale Nahrungsmittelkonzerne wie Nestlé, Unilever oder Cargill an Marktmacht. Menge und Vielfalt der vertriebenen Waren unterliegen dabei einer rasanten Ausweitung und einer permanenten Innovation: So hat zwischen 1960 und 1999 die Zahl der angebotenen Lebensmittel extrem zugenommen. Allein in Deutschland kommen dank eines wettbewerbsbedingt hohen Innovationstempos jedes Jahr etwa 10.000 Lebensmittel neu auf den Markt, von denen viele (60\% aller Backwaren, 40\% der Süßwaren, 38\% der Molkereiprodukte) bereits nach einem Jahr

2 In der Massentierhaltung werden mehr Nahrungsmittel verbraucht als neu geschaffen. Ca. sieben bis zehn Kilogramm Getreide, die dann für die Welternährung nicht mehr zur Verfügung stehen, werden für ein Kilogramm Fleisch verfüttert. Gleichermaßen verfüttern AquaFarmer mehr wild gefangenen Fisch als sie an Zuchtlachs produzieren. Kadaververfütterung, Fischmehl, Hormone, Antibiotika und BSE belegen den Fall der Qualitätsstandards in der Nahrungsmittelproduktion (Schwendter 1995: 234). Dabei geht die Beschleunigung und Kontrolle der generativen Zeiten tierischen und pflanzlichen Wachstums nicht nur zu Lasten der Qualität: der Qualität der Lebensmittel und der Lebensqualität der betroffenen Tiere. Sie geht auch zu Lasten der Umwelt, denn die erzielten Produktivitätssteigerungen sind prinzipiell mit einem hohen Einsatz von Energie und Chemikalien verbunden. Die Landwirtschaft hat somit ihre alte metabolische Bedeutung verloren, durch das Einfangen und die Kontrolle von Solarenergieflüssen wichtigster Energielieferant zu sein. Stattdessen hat sich seit dem Zweiten Weltkrieg in Westeuropa und den USA eine Form der Produktion durchgesetzt, die durch den Rückgriff auf fossile Energien vom Energielieferanten zum Energieverbraucher geworden ist (Bowler 1992). Nur noch 2\% der Bevölkerung Beschäftigung bietend, ist die Landwirtschaft zu einem „bloßen Sektor der Industrie“ (Sieferle 2000: 14) geworden. 
wieder vom Markt verschwunden sind (Tappeser/Baier et al. 1999: 57f). Die Vielfalt der verschiedenen Produkte geht jedoch auf eine relativ kleine Anzahl von Grundprodukten zurück. Durch Standardisierung (Fordismus) und Spezialisierung (Taylorismus) basiert die „Vielfalt“ auf einer „Einfalt“ von wenigen „Monokulturen“ (Weizen, Rind, Schwein etc.) und auf einem Verlust von Agrobiodiversität (Petschow/Idel 2004). Im Zuge der Kombination aus Convenience Food, Functional Food ${ }^{3}$ und Bio-Produkten sind auch Lebensmittel, die in s.g. Naturkostläden angeboten werden, immer mehr zu „Dienstleistungsprodukten" geworden, die einen Mehrfachnutzen in Hinblick auf die schnelle und bequeme Zubereitung, die geschmackliche Qualität und den gesundheitlichen Nutzen versprechen (Mertz 2003).

Mit der industriellen Verarbeitung von Lebensmitteln wird es jedoch nicht nur möglich, den Verderb von Nahrungsmitteln vergleichsweise lange hinauszuzögern, sondern ebenso, den Zubereitungsprozess extrem zu beschleunigen und zu vereinfachen. Aufgrund der Erosion des fordistischen Geschlechtermodells (Männer als „bread winner“ und Frauen als „food server“), der zunehmenden Berufstätigkeit von Frauen und der steigenden Zahl von Single-Haushalten erhöht sich die Nachfrage nach Tiefkühl- oder Fertiggerichten. Von der Konsumseite her wird die Erhöhung der Verarbeitungstiefe aber auch durch einen zunehmenden Verlust an Ernährungswissen und -kompetenz verstärkt: Convenience Produkte werden aus Bequemlichkeit und Zeitgründen gekauft, darüber hinaus, weil vielen KonsumentInnen heute schlicht die Zubereitungskompetenzen fehlen. Nichtregierungsorganisationen wie z.B. Slowfood befürchten, dass Lebensmittelkonzerne und Global Player der Life Style Unternehmen mit ihren Fertiggerichten und Aromastoffen zunehmend Geschmackserwartungen und Ernährungsgewohnheiten definieren und den Trend verstärken, dass im Zeitalter der Mikrowelle, des Convenience Food und der Fast Food Ketten das Wissen über die Zubereitung von Mahlzeiten als kollektives Kulturerbe verloren geht. In welchem Umfang die Nutzung von Convenience Produkten zu einem selbstverständlichen Bestandteil der heutigen Esskultur geworden ist, drückt sich in einem Wahrnehmungswandel aus: Demzufolge wird heute auch das Backen mit Fertigmischungen oder die Zubereitung einer Suppe mit Maggi-Fix überwiegend als eigene Kochleistung definiert (IGLO-Forum-Studie 1995) und nicht mehr als Verlust eigener Kompetenzen verstanden. ${ }^{4}$

3 Dem Interesse an einem gesundheitlichen Nutzen der Ernährung sollen in der Zukunft Functional Food Produkte verstärkt entgegenkommen, die herkömmliche Nahrungsmittel umfassen, denen zusätzlich Anteile an angeblich gesundheitsfördernden Zutaten beigefügt werden: Probiotische Milchprodukte, Getränke mit Vitamin-, Kalzium- oder Eisenzusatz, Brotaufstriche mit cholesterinsenkender Wirkung oder hyppoallergene Babynahrung. Zunehmend werden damit auch Grenzen zwischen Nahrungsmitteln und Medikamenten aufgelöst.

4 Dabei definieren 27\% der Befragten, dass Dosengemüse, mit Butter und Kräutern verfeinert, eine selbstzubereitete Beilage darstellen; ebenso viele finden, dass ein Kuchen auch dann selbstgemacht sei, wenn er aus einer Backmischung unter Zugabe von Eiern und Milch zusammengestellt wird (ebd.). 
Warum ist diese Konstellation aus Massenproduktion, industrieller Verarbeitung und der rapiden Erosion des Wissens über Ernährung problematisch und eine Barriere für Nachhaltigkeitsziele? Da nur ein geringer Prozentsatz der Lebensmittel direkt vom Bauernhof zu den KonsumentInnen gelangt und heute mehr als 95\% der Lebensmittel industriell verarbeitet werden, geraten Landwirte zunehmend in die Funktion des Rohstofflieferanten für die Lebensmittelindustrie. Somit verlieren sie ihre alte agrarische Funktion als direkter Bereitsteller von „Mitteln zum Leben“: Sie produzieren Gemüse für Tiefkühlkost, Tomaten für Ketchup und Tomatenmark, Kartoffeln für tiefgefrorene Pommes Frites und Kartoffelbreipulver, Hähnchen für Chicken McNuggets - Nahrungsmittel, die von den großen Nahrungsmittelkonzernen weiter verarbeitet und beispielsweise als Grundbausteine für die Hamburger, Pizzas und Pastas der großen Fast Food Ketten dienen (Friedmann 1999; Brunner 2002). In der Regel ist die industriell-massenhafte Produktion des Essens mit einem hohen Preisdruck auf die Landwirte verbunden. Abhängig geworden von den Großabnehmern in Lebensmittelindustrie und Einzelhandel können die landwirtschaftlichen Erzeuger die Produktion großer Mengen nur gewährleisten, wenn sie alle Rationalisierungsreserven ausschöpfen, Kunstdünger, Pestizide, Herbizide und Fungizide einsetzen, Flächen zusammenlegen und zum Anbau in Monokulturen übergehen (Hutter 2004). Der Preisdruck zwingt zu Produktionsbedingungen, die einer Orientierung an den Zielen der Nachhaltigkeit durch Umweltschonung und artgerechte Tierhaltung entgegen stehen. ${ }^{5} \mathrm{Ob}$ wohl generell bei wachsender Kontrolle der natürlichen Wachstums- und Reifezeiten und bei zunehmender Verarbeitungstiefe der Stoff- und Energieumsatz steigt, gibt es im Bereich der Convenience Produkte erhebliche Unterschiede in bezug auf die Umweltbelastung, die Qualität der verwendeten Nahrungsmittel und ihren gesundheitlichen Nutzen. Der Preisdruck des Einzelhandels auf die Lebensmittelkonzerne und die Erzeuger zwingt diese jedoch fast immer, möglichst billig zu produzieren und daher z.B. anstelle teurer Rohstoffe den Geschmack durch den Zusatz von Glutamat zu intensivieren und so zu ungewolltem Mehrverzehr zu verführen. Mangelnde Kochkenntnisse und fehlendes Ernährungswissen der KonsumentInnen begünstigen wiederum Fehlernährung und Übergewicht, bestärken eine unkritische Haltung gegenüber Geschmacksverstärkern und Konservierungsstoffen ${ }^{6}$ und fördern die Gleichgültig-

5 Um dem Ziel der Agrarwende - ein ökologisch orientierter Umbau des Ernährungssystems durch die Steigerung ökologisch bewirtschafteter Flächen auf über 20\% - gerecht zu werden, ist der Vertrieb von Convenience Produkten im Naturkosthandel vielfach begrüßt worden, um breitere KäuferInnenschichten zu erreichen. Dabei wird jedoch übersehen, wie problematisch es aus Nachhaltigkeitsperspektive sein kann, wenn sich „Bio-Produzenten der Marktlogik unterwerfen müssen, einem Preisdruck ausgesetzt werden, mehr Verpackungen akzeptieren müssen, zunehmend Fertiggerichte anbieten und Produkte über größere Entfernungen liefern sollen“ (Brunner 2001: 215).

6 Bislang galten chemisch produzierte Aromastoffe als gesundheitlich unbedenklich. Es gibt jedoch in den Ernährungswissenschaften eine Minderheitenposition, wonach Aromastoffe 
keit gegenüber sozialen und ökologischen Standards in der Nahrungsmittelproduktion. An die Stelle von Qualitätsmerkmalen tritt die Ausrichtung von Konsumentscheidungen am Preis sowie an der Bequemlichkeit und Schnelligkeit der Zubereitung.

\subsection{Konsum im Lebensmitteleinzelhandel}

Eine unübersehbare Umwälzung hat nicht nur bei der Produktion und Verarbeitung von Lebensmitteln stattgefunden, sondern auch bei ihrer Distribution und beim täglichen Einkauf: War das Einkaufen in der Nachkriegszeit durch den fast täglichen Besuch vieler kleiner Läden in Wohnungsnähe, den Einkauf im Fachgeschäft des Bäckers, Milchmanns, Gemüsehändlers und Kaufmanns geprägt, so ist es seit der zweiten Hälfte der 1950er Jahre in Westdeutschland zu einer rasanten Verbreitung von „Selbstbedienungsläden“ gekommen. Die Rationalisierung des Einzelhandels durch das Prinzip der Selbstbedienung ist eingebettet in die Verallgemeinerung von Massenfertigung und Massenkonsum im Ernährungsbereich und notwendig, um die Massenkonsumgüter, die nun in der Industrie seriell hergestellt werden, auch massenhaft absetzen zu können. Im fordistischen Wirkungszusammenhang der Produktion von Massenkonsumgütern, der Steigerung der Realeinkommen, der Ausbreitung der Selbstbedienung als Ver- und Einkaufsform und des Massenkonsums (Wildt 1994: 151) werden Vertrieb und Konsum von Lebensmitteln revolutioniert. Parallel zur Ausweitung des Sortiments und zur Diversifizierung der Produkte kommt es durch den Aufbau eines überregionalen Lebensmittelhandels und das Sterben der „Tante-Emma-Läden“ zu einer Vereinheitlichung der Anbieter. Inzwischen hat sich ein dramatischer Konzentrationsprozess im Lebensmitteleinzelhandel vollzogen, der heute fast ausschließlich von großen Handelsketten dominiert wird, die in einer Marktsituation mit hoher Wettbewerbsintensität und geringen Gewinnmargen agieren. Im Kampf um Anteile auf einem Markt, auf dem KundInnen vor allem durch Niedrigpreisstrategien geworben werden, verlangt der Handel nach Produkten, die preisgünstig sind, sich gut transportieren und lagern lassen und in großer Menge geliefert werden können. Durch diese Art der Nachfrage fördert er mit seiner starken Marktmacht als Großabnehmer gegenüber der Lebensmittelindustrie und gegenüber den landwirtschaftlichen Erzeugern letztlich Produktionsstrukturen, die immer wieder Lebensmittelskandale verursacht haben (z.B. durch Fütterung der Tiere mit billigen Importen).

Die Verallgemeinerung der Selbstbedienung als Form der Massendistribution macht es erforderlich, dass die Produkte den KundInnen vorverpackt in Rega-

nicht vorhandene Nahrungsbestandteile wie Fleisch, Obst oder Gemüse vortäuschen, auf die der Körper mit gesteigertem Appetit reagiert und auch bei vollem Magen zum Weiteressen von tatsächlich extrem zucker- und fetthaltigen Nahrungsmitteln anregt. 
len angeboten werden (Wiegemann 1993). In Selbstbedienungsläden ist es kaum denkbar, die Ware, wie in den alten Fachgeschäften, lose verpackt in Kisten, Säcken, Fässern, Paketen oder Korbflaschen anzubieten. Dabei dient die Verpackung im Zusammenhang mit der Renaissance von Markenartikeln, die in den Westdeutschland nach dem Ende der Lebensmittelrationierungen einsetzte, nicht nur als Schutz der Ware, sondern auch als Werbeträger. Die Aufgabe der Verpackung als Erkennungszeichen werbestrategisch etablierter Marken wird immer wichtiger, nicht nur, um als „Vertrauensscharnier“ (Barlösius 1999) Orientierung angesichts eines immer unübersichtlicheren Angebots zu bieten. Noch entscheidender wird für die neuen Selbstbedienungsläden und Supermärkte die Funktion der Verpackung, die Ästhetik der Warenhülle zu einer Projektionsfläche für Wünsche der KäuferInnen zu machen. Wie Waren durch ihre Verpackung mit einem „Gebrauchswertversprechen“ versehen werden, das mit der eigentlichen Beschaffenheit der Ware nichts mehr zu tun hat, und wie Waren mit allen verfügbaren Mitteln zu Marken aufgebaut werden, analysierte W.F.Haug bereits 1973 in seiner Kritik der Warenästhetik. Im Nahrungs- und Genussmittelsektor, der spätestens seit Mitte der 1960er Jahre hochgradig durch Name, Form und Bild des Markenartikels geprägt ist, erhält demnach die Ästhetisierung der Ware eine qualitativ neue Bedeutung und macht den „Schein“ der Ware für den Kaufakt letztlich wichtiger als ihr „Sein“:

„Zunächst bleibt die funktionell bereits abgelöste Gestaltung und Oberfläche, der bereits eigene Produktionsgänge gewidmet werden, mit der Ware verwachsen wie eine Haut. Doch bereitet die funktionelle Differenzierung die wirkliche Ablösung vor, und die schön präparierte Oberfläche der Ware wird zu ihrer Verpackung, die aber nicht wie das bloße Einwickeln als Schutz vor den Gefahren des Transports gedacht ist, sondern als das eigentliche Gesicht, welchselbes statt des Warenleibs der potentielle Käufer zunächst zu sehen bekommt. (...) Nachdem ihre Oberfläche sich von ihr abgelöst hat und zu ihrer zweiten Oberfläche geworden ist, die in der Regel unvergleichlich perfekter als die erste ist, löst sie sich vollends los, entleibt sich und fliegt als bunter Geist der Ware in alle Welt“ (Haug 1973: 60f.).

Nicht nur die farbliche Gestaltung der Verpackungen nach den Vorgaben der Farbenlehre spielt eine strategische Rolle in den Absatzüberlegungen der Lebensmittelindustrie. Auch der Aufdruck von Bildern unberührter Natur (z.B. alte Bauernhöfe oder Tiere auf grünen Wiesen) verleiht den Verpackungen häufig ihr charakteristisches Gesicht und soll der kollektiven Utopie Raum geben, durch den Genuss dieser Lebensmittel mit einem natürlichen Raum und seinen Rhythmen verbunden zu sein - einer unberührten Natur, die nicht von der industrialisierten Welt kontaminiert ist. ${ }^{7}$ Das Spektakuläre an dieser

7 Am Beispiel der Margarine „Rama“ illustriert Wildt (1994) die Naturalisierung industriell erzeugter Lebensmittel in den 1960er Jahren: „Der Versuch, ,Rama’ durch die Goldverpakkung, die Zusätze ,Delikatess' und ,mit dem naturfeinen Geschmack', nicht zuletzt durch den Namen selbst (Rama - Rahm), von der industriellen, synthetischen Produktion auf die Ebene der ,natürlichen', ,guten' Butter zu heben, verleugnete explizit die tatsächliche Substanz, die nach wie vor nichts anderes als Margarine war. Die Verpackung wurde zur Projektionsfläche einer Pluralität von Bedürfnissen, das Signifikat löste sich vom Referenten, jetzt 
Form der Warenästhetisierung ist, dass die affektive Distanz der KonsumentInnen von der Herstellung und Verarbeitung der Lebensmittel im globalen Ernährungssystem auf der Ebene des Konsums durch die Beschwörung eines idealen Naturraums und durch die semiotische Kraft der Warenästhetik wieder aufgehoben wird, indem die tatsächliche industriell geprägte Herkunft der Nahrungsmittel verleugnet wird. Wenn also in Produktion, Verarbeitung und Transport der Lebensmittel alle Abläufe mit dem Ziel ihrer ökonomischen Optimierung kontrolliert und Wachstums- und Reifezeiten in Lebensmittelproduktion und -verarbeitung fast beliebig beschleunigt oder verlangsamt werden, folgt am Ende der Wertschöpfungskette - auf der Ebene des Konsums nämlich - die „Rückkehr zur Natur“: zumindest auf der Warenhülle. Wie erfolgreich die Ästhetik der Warenhülle als Projektionsfläche einer „natürlichen“ Herkunft von Nahrungsmitteln dient, zeigt beispielsweise eine Studie zu „Produktassoziationen beim Kauf von Lebensmitteln“ (IFAV, Köln 2001), die vom Bundesverband der Verbraucherzentralen in Auftrag gegeben wurde (Tschöke 2002). Demzufolge verknüpfen viele VerbraucherInnen mit den Bildern ländlicher Idylle auf Lebensmittelverpackungen Vorstellungen, die mit der Realität der landwirtschaftlichen Massenproduktion nicht oder nur wenig übereinstimmen. Beispielsweise wurden Eier aus Batteriehaltung, die in Kartons mit Abbildungen von Bauernhöfen oder Hühnern angeboten werden, von 17\% der Befragten für Eier aus Freilandhaltung gehalten.

\section{Die Rolle privater Haushalte im Ernährungssystem}

Das heutige Ernährungsangebot ist mit Annehmlichkeiten und Vorzügen für die VerbraucherInnen im Norden verbunden, wird doch saison- und ortsunabhängig ein bezahlbares und ausdifferenziertes Angebot an Nahrungsmitteln aus aller Welt zum Kauf angeboten. Mit der Beschleunigung pflanzlicher und tierischer Wachstumsprozesse wird durch Produktivitätssteigerungen in der

endlich war das Feld frei für jegliche semiotische Codierung“ (ebd.: 176). Als charakteristisches Beispiel für die filmische Werbestrategie der natürlichen Herstellung eines Produktes verweist Karmasin (1999) auf die Kampagne zur Einführung des Fruchtjogurts Landliebe in den 1990er-Jahren: „Im Film wird die Herstellung des Fruchtjogurts gezeigt. Man sieht die Kuh, von der die Milch stammt, sie heißt Lisa, sie trägt einen Blütenkranz und ist die Freundin eines kleinen Mädchens. Die Früchte, die in den Jogurt kommen, werden von einer jungen Bauersfrau oder Sennerin in einem kleinen Hüttchen auf einem Brettchen geschnitten, in kleinen Portionen in den Jogurt gerührt und dann ins Glas gefüllt - das ist Landliebe. (...) niemand glaubt wirklich, dass das Unternehmen, das Landliebe herstellt, Tausende von Sennerinnen beschäftigt oder Gösser, Röster und Schnitter. Man versteht die Filme im Sinne einer ,Als-ob-Behauptung': Landliebe schmeckt so, als ob es von einer Sennerin liebevoll hergestellt und frisch zusammengerührt wurde. Bemerkenswert ist jedoch die Erfindung und Inszenierung dieser Naturwelten insgesamt: Was sie darstellen, ist der Traum von einer Welt, in der die Verhältnisse der modernen Industriegesellschaft, der individualistischen Marktkultur mit ihrer Wertschätzung von Fortschritt, Leistung, Wettbewerb außer Kraft gesetzt sind“ (Karmasin 1999: 277). 
Landwirtschaft der Massenkonsum von Fleisch, Eiern, Getreide, exotischen Früchten und anderen Nahrungsmitteln möglich. VerbraucherInnen profitieren von der Beherrschung der Zukunft, die mit Methoden der Lebensmittelverarbeitung durch Verlängerung der Haltbarkeit oder durch recht exakte Angaben über Verfall und Verderb von Nahrungsmitteln ermöglicht wird. Sie genießen die Vorteile, mit Tiefkühlkost, Konserven oder Importfrüchten den Vorgaben jahreszeitlicher Rhythmen zu entgehen und sparen Zeit, wenn industriell verarbeitete Fertiggerichte nur noch erwärmt werden müssen. Die Rolle der privaten Haushalte im Ernährungssystem verändert sich im Zuge dieser zunehmenden Ubiquität und Omnitemporalität des Ernährungssystems und ist durch eine umfassende Verlagerung von Arbeiten aus den Haushalten hin zum Markt charakterisiert. War das Einkochen von Obst und Gemüse zu Beginn der 1950er Jahre in Deutschland noch eine sehr weit verbreitete Praxis, geht der Zeitaufwand für diese Tätigkeit im Zuge fallender Preise für Konserven in den 1960er und 1970er Jahren drastisch zurück:

„Arbeiten, die ehemals manuell im eigenen Haushalt erledigt wurden, übernahm nun zunehmend die Lebensmittelindustrie. Gemüse musste nicht mehr selbst geputzt werden, sondern war in Dosen kochfertig verfügbar. Geschälte Kartoffeln gab es im Glas, Obstkonserven enthoben die Hausfrau des mühseligen Entsteinens. Die Verwendung von Konserven bedeutete aber zugleich eine wesentliche Veränderung der Konsumpraxis. Die Selbstversorgung verlor ihren Stellenwert für das tägliche Essen, stattdessen nahm die Monetarisierung des Konsums zu“ (Wildt 1994: 131).

Bei der Monetarisierung und Kommodifizierung der Ernährung handelt sich um einen ambivalenten Prozess, der auf der einen Seite Arbeitserleichterungen durch den Konsum von vorgefertigten Nahrungsmitteln in den privaten Haushalten mit sich bringt und Frauen die Vereinbarung von Berufs- und Versorgungsarbeit erleichtert. ${ }^{8}$ Gleichermaßen wird aber die Marktabhängigkeit gesteigert. Die KonsumentInnen verlieren in dieser Struktur direkte Einflussund Gestaltungsmöglichkeiten auf die Produktion und Verarbeitung von Lebensmitteln. Durch die räumliche und zeitliche Trennung von Produktion und Konsum und eine zunehmende Verarbeitungstiefe erleben VerbraucherInnen aber auch eine „affektive Distanz“ von den „Mitteln zum Leben“, die sie essen: Am Ende der globalen Ernährungsketten situiert, ist die Erfahrungswelt

8 Mit der Verlagerung von Arbeiten aus den Haushalten an den Markt geht die Ausweitung weiblicher Beschäftigung in der verarbeitenden Industrie und im Einzelhandel einher. Diesen Prozess haben Goodman und Redclift (1991) mit dem Slogan „Women into Factories, Food into Freezer" plakativ umschrieben - nicht als Ursache-Wirkungs-Verhältnis, sondern als ,interdependence of changes in the labour market and changes in the production and consumption of food commodities" (Goodman/Redclift 1991: 14). Seit den 1970er-Jahren ist durch die Ausweitung von Fastfood-Ketten, ausländischen Spezialitätenrestaurants und Gaststätten der Umfang der Dienstleistungsangebote im Ernährungssektor stark gestiegen. Damit ist ein weiterer Sektor geschaffen worden, in dem die Mehrzahl der Arbeitsplätze von Frauen eingenommen wird. Es handelt sich dabei allerdings in der Regel um prekarisierte Arbeitsverhältnisse, die als Teilzeitarbeitsplätze hochgradig flexibilisiert und im Niedriglohnbereich angesiedelt sind. 
der VerbraucherInnen von den Produktionsorten und Reifezeiten der Nahrungsmittel abgeschnitten und durch ein zunehmendes Nicht-Wissen über Herkunftsort und Saisonalität, über die Arbeits- und Produktionsbedingungen in der Landwirtschaft oder die Zusammensetzung veredelter Produkte geprägt. Eine Vielzahl von Lebensmittelskandalen wie die BSE-Krise oder der Nitrofenskandal haben in den letzten Jahren die Risiken des Ernährungssystems verdeutlicht. Dabei handelt es sich um industriell erzeugte Risiken, die vom Typ her unsichtbar sind, lange unbemerkt wirken und erst dann als Folgen menschlichen Handels sichtbar werden, wenn sie sich in Form von Symptomen materialisieren (Adam 1998). In der Regel ist es nur durch wissenschaftliche Expertenanalysen möglich, eine gesundheitsgefährdende Belastung von Nahrungsmitteln festzustellen. VerbraucherInnen können die Qualität und Sicherheit der Lebensmittel nicht auf der Basis ihres Erfahrungswissens einschätzen.

\section{Wege für einen nachhaltigen Konsum im Ernährungsbereich}

In der Nachhaltigkeitsdebatte bewegt sich der Blick auf die privaten KonsumentInnen zwischen kontrastierenden Polen: Einer Betonung der Abhängigkeit von den vorgekochten Fertiggerichten und den Geschmacksvorgaben der Ernährungsindustrie steht die Einschätzung gegenüber, dass VerbraucherInnen mit ihren Konsumentscheidungen ein hohes Maß an Gestaltungsmacht haben und Umweltbelastungen bereits mit der Nachfrage beginnen. Wahlweise werden KonsumentInnen aufgrund mangelnden Ernährungswissens und fehlender Kochkenntnisse entweder als Unterdrückte einer bevorstehen „Diktatur der Tüte“ (Hutter 2004) angesehen oder als „König Kunde“ für den nachhaltigen Wandel des Ernährungssystems verantwortlich gemacht. Vor dem Hintergrund der vorangegangenen Skizze des Ernährungssystems wird erstens deutlich, dass alle Akteure des Ernährungssystems in Nachhaltigkeitsstrategien einbezogen werden müssen. Dabei gilt es z.B. anzuerkennen, dass es Lebensmittelindustrie und Handelsunternehmen sind, die über Produktinnovationen und Nahrungsmittelzusätze entscheiden und ein dementsprechend hohes Maß an Gestaltungsmacht in diesem Bereich haben. Zweitens ist es notwendig zu fragen, welche Handlungsrestriktionen und -möglichkeiten für private KonsumentInnen in ihren täglichen Konsum- und Ernährungsentscheidungen bestehen, um geeignete Wege für einen nachhaltigen Konsum im Bedürfnisfeld Ernährung zu entwerfen. ${ }^{9}$ Dabei sollen drei Ansätze diskutiert werden, welche die Verän-

9 Dabei wird Nachhaltigkeit in den gängigen Studien mit den Zielen konkretisiert, den Fleischkonsum zu reduzieren und gering verarbeitete Lebensmittel zu bevorzugen. Weiterhin wird Nachhaltigkeit damit assoziiert, Produkte aus ökologischer Landwirtschaft, saisonale und regionale Nahrungsmittel und Produkte aus „fairem Handel“ zu konsumieren. Nachhaltigkeit bezieht jedoch weitere soziale und ökonomische Dimensionen ein, z.B. durch falsche Ernährung verursachte Gesundheitsrisiken zu mindern und zu vermeiden, dass sozial schwache Schichten aufgrund mangelnden Ernährungswissens stärker von ernährungsbedingten Krankheiten betroffen sind als andere (Brunner 2004). 
derung individueller Ernährungspraxen mit grundsätzlichen Umgestaltungen im Verhältnis von Produktion und Konsum resp. der Rolle privater Haushalte im Ernährungssystem verkoppeln. Sie beziehen sich:

- auf die Transformation von strukturellen Rahmenbedingungen, unter denen Kaufentscheidungen getroffen werden,

- auf die Entlastung privater Haushalte durch veränderte Angebotsstrukturen,

- auf das „Empowerment“ privater KonsumentInnen.

\subsection{KonsumentInnen als Bündnispartner für Nachhaltigkeit?}

Um die Entscheidungs- und Gestaltungsmacht von privaten KonsumentInnen zu erhöhen, hat das BMVEL mit großem Aufwand das bundeseinheitliche Bio-Siegel zur Kennzeichnung von Produkten aus kontrolliert biologischem Anbau (nach EU-Verordnung) eingeführt, eine Kampagne zur Information über fair gehandelte Produkte finanziert und immer wieder an die KonsumentInnen appelliert, Nachhaltigkeitskriterien beim Kauf von Lebensmitteln zu berücksichtigen. Die Strategie, die KonsumentInnen für eine Agrar- und Ernährungswende zu gewinnen, basiert zu Recht auf der Betonung von Entscheidungsund Gestaltungsmöglichkeiten der VerbraucherInnen, läuft aber immer auch Gefahr, individuelle Konsumentscheidungen zu moralisieren und Handlungsmöglichkeiten bzw. -restriktionen der KonsumentInnen und die strukturell angelegten Barrieren für eine Veränderung von Ernährungsmustern im Sinne einer Agrar- und Ernährungswende nicht ausreichend zu berücksichtigen: Dazu gehört, wie seit Beginn der Nachhaltigkeitsdebatte betont wird, dass die Preissignale für den Konsum (langfristige) ökologische Kosten externalisieren und die Ubiquität des Ernährungssystems auf dem Konsum von Waren aus aller Welt basiert, deren Preise nicht „die ökologische Wahrheit“ sagen (Spangenberg/Lorek 2001). Denn preislich gesehen spielen die Distanzen keine Rolle und ökonomisch betrachtet sind die größeren Entfernungen, die in den Mahlzeiten automatisch mit gegessen werden, unsichtbar. ${ }^{10} \mathrm{Da}$ der Konsum im Ernährungssystem durch die affektive Distanz und die räumliche und zeitliche Entkoppelung von Produktion und Konsum geprägt ist, werden individuelle Kaufentscheidungen in modernen Supermärkten und Discountern durch Preissignale, aber auch durch die Bilder der Werbewelt beeinflusst, welche die unmittelbare Erfahrungswelt im Konsumgeschehen prägen und das Produktimage geschickt mit unbewussten Wünschen (in Bezug auf Prozesse der Identitätsbildung oder der sozialen Distinktion) verknüpfen. Eine „Reno-

10 Der Lebensmittelkonsum Deutschlands ist in den letzten 30 Jahren nur leicht angestiegen (gemessen in Kilogramm pro Kopf), der Transportaufwand hat sich jedoch im Zeitraum von einer Generation fast verdoppelt. Obwohl also bei den Mahlzeiten größere Entfernungen „mitverzehrt werden“, sind die Nahrungsmittelausgaben prozentual gesunken (Böge/von Winterfeld 1995: 110). 
vierung dieser Bilderwelt“ fordern Verbraucherorganisationen z.B. im Streit um die Vermarktung von Eiern aus Käfighaltung mit Bildern von frei laufenden Hühnern und alten Bauernhöfen. Sie wollen eine Novellierung des Wettbewerbsrechts, das veraltet und nur anbieterorientiert sei, indem es Abbildungen nicht als „relevante Informationen“, sondern auch als „Gestaltungselemente“ definiere. In die gleiche Richtung zielt die Forderung, alle Inhaltsstoffe zu deklarieren und Auskunft über die durchgeführten Produktionsprozesse zu geben, um den VerbraucherInnen eine ausreichende Entscheidungsgrundlage zu bieten und eine größere Wahlfreiheit zu gewährleisten. ${ }^{11}$ Nicht zuletzt ist der Konsum von Nahrungsmitteln von Ernährungsmustern geprägt, die auf sozial unterschiedliche Lebensstile zurückgehen und eine milieuspezifische Verschiedenheit der Geschmäcker spiegeln. Neuere Nachhaltigkeitsansätze zielen darauf $a b$, in Veränderungsstrategien für einen nachhaltigeren Konsum an sozial differenzierte Ernährungsstile anzuknüpfen, auf Motivlagen der Betroffenen einzugehen (z.B. stärkere Gesundheitsorientierung) und darauf abgestimmte Umstellungsvorschläge zu entwickeln (Empacher 2004):

„Geschmäcker lassen sich aber nicht so leicht verändern. Ernährungsbezogene Nachhaltigkeitsstrategien müssen dies berücksichtigen und in Rechnung stellen, dass (...) eine nachhaltigere Lebens- und Ernährungsweise ohne Überforderung der Individuen nur in vielfältigen Kompromissbildungen vor sich gehen kann" (Brunner 2002: 260)

Zieht man eine Bilanz der Handlungsmöglichkeiten und -restriktionen der KonsumentInnen im Machtgefüge des Ernährungssystems, dann zeigt sich, dass für einen tiefgreifenden Wandel des Konsums im Ernährungsbereich nicht nur eine Veränderung individueller Kaufentscheidungen notwendig ist, sondern auch die Anreizsysteme und Kontexte, in denen Kaufentscheidungen getroffen werden, in die Umgestaltung einbezogen werden müssen. Sonst bleiben KonsumentInnen prekäre Bündnispartner auf dem Weg in ein nachhaltigeres Ernährungssystem und beharren mit ihren Konsumentscheidungen auf einem Ernährungssystem, das - wie im Schlaraffenland - eine breite Palette an Nahrungsmitteln zur Verfügung stellt, in dem aber die sozialen und ökologischen Kosten mit den Bildern der Werbewelt verdeckt sind und Risiken nur in Form kurzfristiger Skandalisierungen aufbereitet werden, um dann wieder aus dem Kurzzeitgedächtnis der VerbraucherInnen gestrichen zu werden.

\subsection{Eine nachhaltige Kantinenkultur}

Nicht zuletzt im Kontext der Debatte um Übergewicht bei Kindern ist immer wieder an „Familien“ appelliert worden, regelmäßige Mahlzeiten mit frisch verarbeiteten Lebensmitteln anzubieten und so eine nachhaltige Esskultur (u.a.

11 Die Ausrichtung von Konsumentscheidungen an Produktinformationen erfordert jedoch entsprechende kognitive Kompetenzen im Deuten von Inhaltsangaben und die Bereitschaft, hierfür Zeit aufzuwenden. 
an den Zielen Gesundheit und Umweltverträglichkeit orientiert) zu fördern. Mit dem Übergang in ein postfordistisches Ernährungssystem ist jedoch das Phänomen einer zunehmenden „Entrhythmisierung der Mahlzeitenordnung“ zu beobachten. Im Zuge der Flexibilisierung von Arbeitszeiten und der zunehmenden Erwerbstätigkeit von Frauen verliert die bürgerliche Mahlzeitenordnung, die auf einem gemeinsamen Frühstück, Mittag- und Abendessen im Kreis der Familie basierte, an Bedeutung. Weil mit der Erosion des fordistischen Hausfrau-Ernährer-Modells "Zeitpuffer" in den Familien durch nicht erwerbstätige Frauen wegfallen und die Individualisierung der Zeitmuster in den Familien zunimmt, wird es schwieriger, gemeinsame Rhythmen für Familienmahlzeiten zu finden (Schlegel-Matthies 2002). Hinfällig wird dadurch die Vorstellung einer vermeintlich unbegrenzt und kostenlos zur Verfügung stehenden Ressource Versorgungsarbeit, welche in den Appellen an gesunde Ernährung und regelmäßige gemeinsame Mahlzeiten häufig implizit steckt. In vielen privaten Haushalten wird mit dem gesellschaftlichen Wandel durch die Feminisierung und Flexibilisierung der Beschäftigung und der zunehmenden Zahl allein Lebender die Zeit für Versorgungsarbeit knapper. Die Rolle der Haushalte als „elastische Zeitressource“ (Elson 1992) geht zunehmend verloren. Die Frage ist, wie angesichts des Verlustes „informeller Flexibilitätsreserven" in den Familien eine zukunftsfähige Organisation von Versorgungsarbeit im Ernährungsbereich aussehen kann (Vinz 2005). Im Sinne der Geschlechtergerechtigkeit als einer zentralen Norm nachhaltiger Entwicklung sind ökologische Ent- und zeitliche Belastungen im Zusammenhang mit der unterschiedlichen Verteilung zwischen den Geschlechtern zu thematisieren und Nachhaltigkeitsstrategien zu finden, welche eine „Privatisierung und Feminisierung von Ernährungs- und Umweltverantwortung" vermeiden. Daher ist es notwendig, Organisationsformen der Versorgungsarbeit zu finden, welche die Last der Veränderung nicht allein den KonsumentInnen überlassen, sondern mit einer Veränderung der Angebotsstrukturen die Ausgangsbasis für veränderte Praxen der Ernährung zu schaffen (Brunner 2004). Für die Förderung einer nachhaltigen Esskultur sind dementsprechend Nachhaltigkeitsallianzen im Bereich der institutionellen Gemeinschaftsverpflegung und ein Ausbau von Kantinen und Mensen für Schulen, Kinderbetreuungseinrichtungen oder auch Betrieben notwendig. Ein Schub für die Stärkung einer nachhaltigen Kantinenkultur in Deutschland könnte sich beispielsweise aus dem Vorhaben der Bundesregierung ergeben, im Rahmen des Investitionsprogramms „Zukunft Bildung und Betreuung“ 10.000 neue Ganztagsschulen aufzubauen. Um die Chance nicht zu versäumen, hohe Qualitätsstandards in Bezug auf die Ernährung und Nachhaltigkeit zu verankern, warnt der DGE-Arbeitskreis „Ernährung und Schule“ davor, „Schulverpflegung ausschließlich bzw. vorrangig unter ökonomischen Gesichtspunkten auszuwählen“ (Arbeitskreis „Ernährung und Schule“ (DGE) 2003; Bundesministerium für Bildung und Forschung 2003) und den Angebo- 
ten der Schnellrestaurant-Ketten und der Catering Services nachzugeben, die derzeit durch Deutschland reisen und die Schulen mit günstigen Vertragsabschlüssen zu ködern versuchen. Dementsprechend hat auch das BMVEL ein mehrjähriges Schwerpunktprogramm gestartet, um Ernährungsaufklärung und -bildung an den Schulen zu unterstützen und die Qualität der Mittagsverpflegung an Ganztagsschulen zu sichern (Wollersheim 2004). Dabei steht allerdings die Orientierung an Kriterien einer vollwertigen und gesunden Auswahl von Nahrungsmitteln stärker im Vordergrund als die Berücksichtigung regional oder ökologisch produzierter Produkte. Wie weit die Nachhaltigkeit der Gemeinschaftsverpflegung im Hinblick auf die Umweltqualität und den Gesundheitswert der verwendeten Nahrungsmittel schwanken kann, zeigt das Beispiel der USA, wo die Schulen an den Vertrieb der großen Getränkehersteller (Cola, Fanta etc.) gebunden sind und gedrängt werden, „den Schülern unbegrenzt Zugang zu den Automaten zu gewähren und es den Schülern zu erlauben, im Klassenzimmer Cola zu trinken“ (Moore 2002: 145). Mit dem Aufbau von Ganztagsschulen bietet sich in Deutschland nun die Perspektive, dem Ziel der Agrarwende entsprechend den Konsum von regional oder biologisch produzierten Nahrungsmitteln zu fördern und entsprechende Mahlzeitenangebote in den Schulkantinen auch finanziell zu unterstützen. Die Ausweitung einer an Nachhaltigkeitskriterien orientierten Mittagsverpflegung an Ganztagsschulen erfasst jedoch nur einen kleinen Teil der Bevölkerung. Mit dem hegemonialen Konzept der Halbtagsschule wird jedoch noch immer vorausgesetzt, dass Eltern mittags Zeit haben, für ihre Schulkinder zu kochen. Welche Auswirkungen die Hartz-Reformen, die Unterfinanzierung von öffentlichen Halbtagsschulen oder der Mangel an Kinderbetreuungseinrichtungen auf das Ernährungsverhalten von Kindern haben, steht auf einem anderen Blatt.

\subsection{Empowerment von KonsumentInnen}

Eine sehr viel tiefer greifende Neuorientierung in der Produktentwicklung und -gestaltung basiert auf dem Umbau asymmetrischer Machtverhältnisse zwischen Herstellern und KonsumentInnen. Mit dem Vorschlag für ein „technologisches Empowerment“" wird in der Nachhaltigkeitsdebatte eine Strategie für den Weg zur nachhaltigen Gestaltung von Produkten und Stoffen entworfen (Schultz 2001; Weller 2004) $^{12}$. Um die Gestaltungsmacht der KonsumentInnen durch Formen direkter Demokratie zu erhöhen, wird beispielsweise gefordert, sie an Entwicklungs- und Gestaltungsprozessen zu beteiligen, ihre Alltagskenntnisse gegenüber dem Wissen von technischen Experten als gleichwertig

12 (Technologisches) Empowerment von KonsumentInnen ist nach Schultz (2001) und Weller (2004) integraler Bestandteil feministischer Nachhaltigkeitsstrategien und notwendig, um Machtasymmetrien zwischen Produktion und weiblich konnotiertem Konsumbereich aufzubrechen. 
anzuerkennen und so eine verbesserte Produktgestaltung - orientiert an unterschiedlichen Alltagserfordernissen - zu ermöglichen. Dies korrespondiert mit der Forderung, die Alltagsperspektive in Ernährungspolitik und -forschung besser zu integrieren und, als Voraussetzung für eine erfolgreiche Nachhaltigkeitskommunikation, die primär naturwissenschaftlichen Perspektiven der Ernährungswissenschaft mit konkreten gesellschaftlichen Ernährungsverhältnissen zusammen zu denken (Rehaag/Waskow 2004). Nach Weller (2004) könne nur im Rahmen eines „technologischen Empowerments“ z.B. durch direkte Mitsprache in der Produktentwicklung und -gestaltung die Gestaltungsmacht der EndverbraucherInnen tatsächlich erhöht und der partizipative Anspruch des Nachhaltigkeitskonzepts erfüllt werden.

Wie allerdings ein Empowerment der KonsumentInnen im Ernährungsbereich konkret aussehen soll, ist bisher eine Leerstelle in der Nachhaltigkeitsforschung: Gibt es im Sinne der Qualitätssicherung Interessenallianzen zwischen Produzenten und NutzerInnen? Ist die verarbeitende Industrie bereit, für partizipative Verfahren zusätzliche Kosten und Zeitaufwand zu akzeptieren, oder ist der Spielraum für technologisches Empowerment durch marktbedingt immer kürzer werdende Innovationsrhythmen zu klein? Weller geht mit dem Empowerment-Konzept noch weiter, wenn sie darüber hinaus fordert, dass Produktentwicklung und -gestaltung auch die „Reproduktionsfähigkeit von Natur und Gesellschaft" berücksichtigen und eine vollständige Information der KonsumentInnen gewährleisten müsste. Diese Forderung birgt Konflikte: Bisher war es weder durchsetzbar, dass die Lebensmittelindustrie eine vollständige Transparenz über Produktionsprozesse und die verwendeten Stoffe herstellt, noch möglich, einen gesellschaftlichen Konsens zu finden, die Herstellung und den Konsum von Nahrungsmitteln wie Fleisch mit hoher Umweltbelastung zu reduzieren oder eine breite Akzeptanz für Preiserhöhungen von Nahrungsmitteln herzustellen, die unter Einhaltung von sozialen und ökologischen Standards produziert werden. Sollen Fragen dieser Art durch Ermächtigung der KonsumentInnen im Rahmen partizipativ angelegter Prozesse mit den Herstellern verhandelt werden? Und sind nur zukünftige NutzerInnen (mit hoher Kaufkraft) an der Demokratisierung von Produktentscheidungen zu beteiligen oder auch diejenigen, die von Umweltfolgen westlicher Produktions- und Konsummuster (z.B. durch Klimawandel) negativ betroffen sind? Wenn die moderne Konsumgesellschaft aufgrund der globalen ökologischen Krise an ihre Grenzen gelangt und sich grundlegend verändern muss, um Bestand zu haben, bleibt offen, ob ein „technologisches Empowerment“ diesen Transformationsprozess vorantreiben kann. In der „Plattform für Ernährung und Bewegung e.V.“, die vom Bundesministerium für Verbraucherschutz, Ernährung und Landwirtschaft (BMVEL) gegründet wurde, um der Zunahme von Übergewicht bei Kindern und Jugendlichen entgegenzuwirken, sind KonsumentInnen als „ExpertInnen des Alltags“ nicht einbezogen. Dafür 
beteiligen sich neben Krankenkassen, dem Sportbund, Gewerkschaften, KinderärztInnen durch finanzielle Unterstützung und aktive Mitarbeit auch der Bund für Lebensmittelrecht und Lebensmittelkunde e.V. (BLL) als Vertretung der Lebensmittelindustrie. Deren Beteiligung an dem Fonds war die Drohung von Verbraucherministerin Künast vorangegangen, die Ernährungsindustrie zu Zwangszahlungen in einen Fonds für eine bessere Ernährungsaufklärung zu verpflichten. Verbraucherorganisationen wie z.B. Foodwatch (2004) kritisieren zum einen, dass in dem Gremium die Vertreter der Lebensmittelindustrie sitzen und sich selbst kontrollieren sollen. Sie bemängeln zum anderen jedoch eine einseitige Schuldzuweisung an die Industrie, mit der ausgeblendet werde, dass dicke Kinder überproportional häufig in sozial schwachen Familien zu finden seien. Die Zukunft der Kinderernährung hänge vielmehr auch von den Auswirkungen der Hartz IV Reformen ab und sei durch den Mangel bezahlbarer Betreuungsmöglichkeiten mit einer guten Mittagsverpflegung bedingt. Die Frage ist, ob eine Auseinandersetzung mit den Betroffenen durch Partizipation ganz andere Folgen für die Politikgestaltung haben würde als die Organisation von Koch- und Sportkursen und den Blickpunkt auf Fragen der Finanzierung nachhaltigen Konsums für sozial Schwächere richten würde.

Für die Förderung nachhaltigen Konsums und zukunftsfähiger Ernährungsstile ist eine Ernährungspolitik erforderlich, die als integratives Konzept Umweltund Agrarpolitik mit Bildungs-, Familien-, Sozial- und Gesundheitspolitik verknüpft. An die Stelle einer Moralisierung von Kaufentscheidungen und einer „Privatisierung und Feminisierung von Umweltverantwortung“ (Weller 2004) tritt die Notwendigkeit, die Rahmenbedingungen, in denen Kaufentscheidungen getroffen werden, zu verändern, alltagsangepasste Angebotsstrukturen zu schaffen und die Gestaltungsmacht privater KonsumentInnen durch Partizipation und Ernährungsbildung zu erweitern.

\section{Literatur}

Adam, Barbara (1998): Timescapes of modernity. The environment and invisible hazards, London/New York.

Arbeitskreis „Ernährung und Schule“ (DGE) (2003): DGE fordert klare Regelungen zur Qualität des Mittagessens für die Ernährung in Ganztagsschulen, in: DGE-aktuell 4.

Barlösius, Eva (1999): Soziologie des Essens: eine sozial- und kulturwissenschaftliche Einführung in die Ernährungsforschung, Weinheim.

BMVEL (2004): Aufklärungskampagne zum nachhaltigen Konsum „Echt gerecht - clever kaufen“, Grußwort der Bundesministerin Renate Künast, www.echtgerecht.de.

Böge, Stefanie/von Winterfeld, Uta (1995): Aus dem Rhythmus? Über den Konsum von Lebensmitteln in zeitlicher und räumlicher Perspektive, in: Zeit-Fraß. Zur Ökologie der Zeit in Landwirtschaft und Ernährung, Politische Ökologie, Sonderheft Nr. 8.

Bowler, Ian (1992): The industrialization of agriculture, in: Bowler, Ian (Ed.): The Geography of Agriculture in Developed Market Economies, New York: 7-31.

Brunner, Karl-Michael (2001): Zukunftsfähig essen. Kommunikation über Nachhaltigkeit am Beispiel des Handlungsfeldes Ernährung, in: Fischer, Andreas/Gabriela Hahn (Hrsg.): Vom schwierigen Vergnügen einer Kommunikation über die Idee der Nachhaltigkeit, Frankfurt a.M.: 207-228. 
Brunner, Karl-Michael (2002): Menüs mit Zukunft: Wie Nachhaltigkeit auf den Teller kommt oder die schwierigen Wege zur gesellschaftlichen Verankerung einer nachhaltigen Ernährungskultur, in: Scherhorn, Gerhard/Christoph Weber (Hrsg.): Nachhaltiger Konsum. Auf dem Weg zur gesellschaftlichen Verankerung, München: 257-268.

Brunner, Karl-Michael (2004): Nachhaltige Ernährung: Das ganze Ernährungssystem ist gefordert!, Monatsthema September 2004: Nachhaltiger Konsum und zukunftsfähiges Essen, www.nachhaltigkeit.at.

Bundesministerium für Bildung und Forschung (2003): Keine Chance den Fritten, www.ganztagsschulen.org/593.php?print $=1$.

Empacher, Doris/Claudia (Hrsg.) (2004): Ernährung anders gestalten, München.

Fine, Ben/Heasman, Michael/Wright, Judith (1996): Consumption in the Age of Affluence. The World of Food, London/New York.

Foodwatch (2004): Dünne Diskussion um „dicke Kinder“, www.foodwatch.de.

Friedland, William H. (1994): The New Globalization: The Case of Fresh Produce, in: Bonanno, Alessandro et. al. (Ed.): From Columbus to ConAgra. The Globalization of Agriculture and food, Lawrence, Kansas: 173-189.

Friedland, William H. (1994a): The Global Fresh Fruit and Vegetable System: An Industrial Organization Analysis, in: McMichael, Philip (Ed.): The Global Restructuring of Agro-Food Systems, Ithaca/London: 173-189.

Friedmann, Harriet (1999): A Sustainable World Food Economy, in: Keil, Roger (Ed.): Political Ecology: global and local, London/New York: 87-101.

Goodman, David/Redclift, Michael (1991): Refashioning Nature. Food, Ecology and Culture, London/New York.

Haug, Wolfgang Fritz (1973): Kritik der Warenästhetik, Frankfurt a.M.

Hutter, Claus-Peter (2004): Das Volk der Tütenkocher. Wie Fehlernährung Bürger und Landschaft krank macht, in: Das Parlament, 54. Jg., Nr. 26: 3.

IGLO-Forum-Studie (1995): Genussvoll essen, bewusst ernähren - Gemeinsamkeiten am deutschen Tisch, Hamburg.

Kaller-Dietrich, Martina (2004): Über den Tellerrand hinaus: Zur Globalisierung der Ernährung, in: iz3w.informationszentrum 3. welt 278/279 (August/September 2004): 31-33.

Karmasin, Helene (1999): Die geheime Botschaft unserer Speisen. Was Essen über uns aussagt, München.

König, Wolfgang (1998): Fast Food. Zur Ubiquität und Omnitemporalität des modernen Essens, in: Adam, Barbara/Karl-Heinz A. Geißler/Martin Held (Hrsg.): Die Nonstop-Gesellschaft und ihr Preis, Stuttgart: 45-62.

Leicht-Eckardt, Elisabeth (1999): Ausstattungsvarianten und Nutzungsformen von Küchen vom Achtzehnten Jahrhundert bis heute, in: Miklautz, Elfie/Herbert Lachmayer/Reinhard Eisendle (Hrsg.): Die Küche. Zur Geschichte eines architektonischen, sozialen und imaginativen Raums, Wien/Köln/Weimar: 161-206.

Mertz, Torsten (2003): Zwischen Techno-Food und Ökolandbau. Die Ess-Klasse der Zukunft, in: Politische Ökologie 73/74.

Moore, Michael (2002): Stupid White Men. Eine Abrechnung mit dem Amerika unter George W. Bush, München.

Petschow, Ulrich/Idel, Anita (2004): Das globale Huhn, in: PROKLA. Zeitschrift für kritische Sozialwissenschaft Heft 135, 34. Jg., 2004, Nr. 2: 263-285.

Prahl, Hans-Werner/Setzwein, Monika (1999): Soziologie der Ernährung, Opladen.

Rehaag, Regine/Waskow, Frank (2004): Zur Emanzipation der Ernährungspolitik. Die BSE-Krise als Chance für eine Wende, in: Öko-Mitteilungen 4/2004: 16-18.

Schlegel-Matthies, Kirsten (2002): Die Tischgemeinschaft vor dem Aus? „Liebe geht durch den Magen" Mahlzeit und Familienglück im Wandel der Zeit, in: Bürger im Staat: Nahrungskultur, Essen und Trinken im Wandel 4/ 2002.

Schneider, Manuel (1999): Turbotiere, in: Politische Ökologie 57/58 (Januar/Februar 1999): 76-78.

Schultz, Irmgard (2001): Der blinde Fleck zwischen Politik und Technikwissenschaften. Strategien eines scientific-technological empowerment als Perspektive feministischer Wissenschaft und Politik, in: femina politica 2/2001: 116-128. 
Schwendter, Rolf (1995): Arme essen, Reiche speisen. Neuere Sozialgeschichte der zentraleuropäischen Gastronomie, Wien.

Sieferle, Rolf-Peter (2000): Im Einklang mit der Natur, in: Baumunk, Bodo-Michael/Jasdan Joerges (Hrsg.): 7 Hügel - Bilder und Zeichen des 21. Jahrhunderts. Dschungel (Band II), Berlin: 12-16.

Spangenberg, Joachim H./Lorek, Sylvia (2001): Sozio-ökonomische Aspekte nachhaltigkeitsorientierten Konsumwandels, in: Aus Politik und Zeitgeschichte B 24/2001: 24-29.

Tansey, Goff/Worsley, Tony (1995): The Food System. A Guide, London.

Tappeser, Beatrix/Baier, Alexandra/Dette, Birgit, et al. (1999): Die blaue Paprika. Globale Nahrungsmittelproduktion auf dem Prüfstand, Basel/Boston/Berlin.

Tschöke, Alice (2002): Werbung mit ländlicher Idylle: Bundesverband der Verbraucherzentralen führt Musterprozess, WDR Fernsehen, ServiceZeit Kostprobe, in: http://www01.wdr.de/tv/ service/kostprobe/inhalt/20020121/b_5phtml?druck=1.

Vinz, Dagmar (2005): Zeiten der Nachhaltigkeit. Perspektiven für eine ökologische und geschlechtergerechte Zeitpolitik, Münster, im Erscheinen.

Weller, Ines (2004): Nachhaltigkeit und Gender. Neue Perspektiven für die Gestaltung und Nutzung von Produkten, München.

Wiegemann, Karlheinz (1993): Supermarkt und Emmaladen: aus der Geschichte der Warenverpackung, 1993-94, Begleitbuch zur gleichnamigen Wanderausstellung des Westf. Museumsamtes, Münster.

Wildt, Michael (1994): Vom kleinen Wohlstand. Eine Konsumgeschichte der fünfziger Jahre, Frankfurt a.M.

Wollersheim, Regina (2004): Fit und gesund durch ausgewogene Ernährung, in: ÖkoMitteilungen 4/2004: 18-19.

\section{Verlag WESTFÄLISCHES DAMPFBOOT}

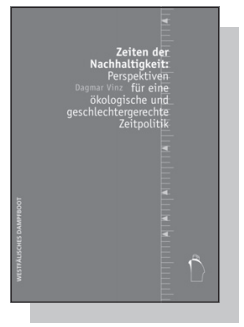

Dagmar Vinz

Zeiten der Nachhaltigkeit: Perspektiven für eine ökologische und geschlechtergerechte Zeitpolitik

2005 - 280 Seiten - € 29,80

ISBN 3-89691-613-0

Hafenweg 26a / D-48155 Münster

Tel.: 02513900480 / Fax: 025139004850 info@dampfboot-verlag.de/www.dampfboot-verlag.de

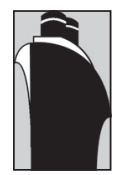




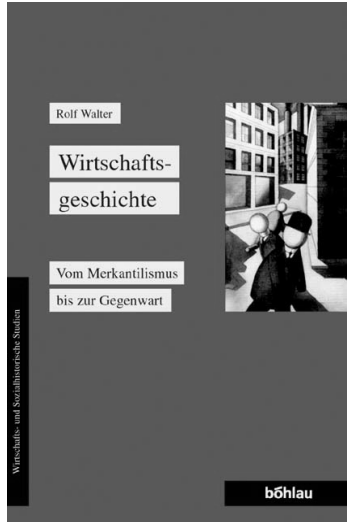

\section{Rolf Walter \\ Wirtschaftsge- schichte \\ Vom Merkantilismus \\ bis zur Gegenwart \\ (Wirtschafts- und Sozial- \\ historische Studien, \\ Band 4)}

Das für die 4. Auflage gründlich überarbeitete und aktualisierte Buch erschließt in leicht lesbarer Form die deutsche Wirtschaftsgeschichte vom Zeitalter des Merkantilismus bis zur Gegenwart. In elf chronologisch aufeinanderfolgenden Kapiteln werden die wesentlichen Grundzüge der Wirtschaftsgeschichte in diesem Zeitraum strukturiert und prägnant dargelegt. Die Darstellung bietet einen umfangreichen Stoff gerafft und selektiv dar. Jedem Kapitel folgen zur Vertiefung und Ergänzung die wichtigsten Literaturempfehlungen sowie eine Reihe von Kontroll- und Wiederholungsfragen.

Das Werk enthält eine umfangreiche Bibliographie und ein ausführliches Register. Als Lehr- und Studienbuch richtet es sich vorwiegend an Studenten und Lehrer in den Fächern Wirtschafts- und Sozialgeschichte, Volkswirtschaft, Betriebswirtschaft und Geschichte.

4. überarbeitete und

aktualisierte Auflage 2003.

XVI, 357 Seiten. 27 s/w-Abb.,

zahlreiche Grafiken und

Karten. Broschur.

$€ 24,90 / S F r$ 42,-

ISBN 3-412-11803-6

Ursulaplatz I, D-50668 Köln, Telefon (o22I) 9I 39 oo, Fax 9I 39 oir 